\title{
Quand les recherches en sciences sociales s'invitent dans la gestion de l'eau
}

\author{
Fabienne Barataud $^{1, *}$, Florence Hellec ${ }^{2}$, Alix Levain $^{3}$ et Sandrine Petit ${ }^{4}$ \\ 1 Géographe, Inra, Aster Mirecourt, Mirecourt, France \\ 2 Sociologue, Inra, Aster Mirecourt, Mirecourt, France \\ 3 Socioanthropologue, CNRS, UMR6308 Amure, Plouzané, France \\ ${ }^{4}$ Géographe, Inra, AgroSup Dijon, Université Bourgogne Franche-Comté, UMR1041 Cesaer, Dijon, France
}

Reçu le 18 août 2017. Accepté le 2 mars 2018

La participation de chercheuses en sciences sociales dans un projet engageant un lien étroit avec des usagers ou des gestionnaires sur un territoire n'est jamais acquise. C'est ce travail de négociation ou de repositionnement constant qu'explorent ces quatre expériences, singulières et complémentaires à la fois, à partir d'un retour réflexif des auteurs sur leurs pratiques de recherche en société. Entre dispositifs de recherche et d'action publique, des liens, parfois invisibles, se construisent peu à peu, proposant de nouvelles questions ou des réajustements qui participent d'une démarche interdisciplinaire.

La Rédaction

Résumé - La gestion de la qualité de l'eau est soumise à deux injonctions : un impératif scientifique et un impératif participatif. Au travers de notre expérience de chercheuses en sciences sociales engagées dans quatre démarches de protection associant des projets de recherche souvent pluridisciplinaires, cet article propose un retour réflexif sur les attentes exprimées par rapport à notre participation, ainsi que sur l'évolution de notre positionnement. Il apparaît alors que la place des chercheurs en sciences sociales n'est jamais totalement acquise, parce qu'elle produit des connaissances essentiellement dialogiques, dont les objets se déplacent et se transforment au fil des enquêtes et des interactions avec les porteurs d'enjeux. C'est finalement moins par l'intervention directe que par la réorientation de nos questions de recherche vers des problématiques liées à ces enjeux que nous avons pu partiellement contribuer aux dispositifs de gestion des pollutions diffuses d'origine agricole.

Mots-clés : agriculture / environnement / sciences sociales / pollutions diffuses / gestion de l'eau

Abstract - When social scientists involve themselves in water management. Two major imperative, that potentially constitute a double bind, tend to frame the management of non-point source pollutions due to farming activities in Western Europe today: a scientific imperative due to persisting biophysical uncertainties and their social use; and a participative one. As social scientists involved in four water quality management programs in rural areas between 2010 and 2014 the authors were all caught up in a complex situation, i.e., the need to develop social knowledge and to take into account the lack of legitimacy of social science compared to biophysical science when dealing with complex environmental issues on the one hand, and the timing and frame of public policies on the other. This article builds upon their experience: through a cross-cutting analysis, it highlights common features beyond the diversity of local configurations and the expectations of stakeholders regarding science in general, and social science in particular. No direct change in the situation has been observed in the short term. Meanwhile, reflexivity and the very dialogic nature of social knowledge progressively re-shapes research objects and questions.

Keywords: farming / environment / social science / non-point source pollution / water management

\footnotetext{
*Auteur correspondant : fabienne.barataud@inra.fr
} 
«Je t'aime bien. Mais j'aimerais mieux que l'Inra m'envoie des agronomes. Pas une sociologue.»

Un président de commission locale de l'eau, mai 2011.

Pour progresser vis-à-vis des enjeux de restauration de la qualité des eaux, de quelles connaissances a-t-on besoin? L'interlocuteur cité en épigraphe a tranché! Pourtant, les sciences sociales jouent bien un rôle dans la mise en œuvre des politiques de l'eau, par la compréhension des problèmes, des jeux d'acteurs qui permettent ou non le changement, et la révélation des points aveugles d'une situation (Narcy, 2013). Parmi les scientifiques, les chercheurs en sciences sociales occupent une place peu visible alors que leurs travaux ont dévoilé des failles dans les dispositifs de concertation et de décision de gestion de l'eau : manque de moyens en temps et en expertise pour certains participants (Brun, 2011), terminologie complexe, personnes exclues du périmètre territorial, planification par schémas directeurs (Bouleau et Richard, 2008). C'est sur la place de ces chercheurs dans les dispositifs participatifs et dans la production de connaissances autour de la gestion de l'eau que porte le présent article.

En France, comme dans la plupart des pays d'Europe de l'Ouest, l'agriculture et les agriculteurs sont interpellés en raison de pollutions diffuses aux nitrates et au phosphore (Parris, 2011). Mais les pollutions diffuses qui affectent la qualité des eaux souterraines et de surface sont aussi des pollutions confuses : d'une part parce que les flux de polluants restent difficiles à tracer, laissant une large place aux incertitudes (Raadgever et al., 2011); d'autre part parce que les rôles des usagers, gestionnaires, chercheurs et experts impliqués dans leur gestion sont entremêlés et changeants. Cette situation génère de nombreuses controverses, que ce soit au sujet de la responsabilité des acteurs, du caractère opérationnel des solutions envisagées, ou encore de la résilience des systèmes et les délais de réponse des écosystèmes aux changements de pratiques.

Dans tous les cas, progresser sur les objectifs de qualité d'eau requiert des formes de coopération (Barraqué et Viavattene, 2009) ou des négociations complexes dans lesquelles plusieurs groupes d'intervenants disposent d'une part de légitimité. Les chercheurs en sciences biophysiques sont alors convoqués pour délivrer des connaissances sur les flux d'éléments physiques et les origines des pollutions qui dégradent la qualité de l'eau, le contexte de la directive cadre sur l'eau accentuant les besoins de connaissances pour mettre en place une gouvernance territorialisée de la qualité de l'eau (Steyaert et Ollivier, 2007). Ainsi, la gestion de la qualité de l'eau, telle que nous l'avons observée sur quatre terrains, est soumise à deux injonctions : un impératif participatif (Blondiaux et Sintomer, 2009) et un impératif scientifique et technique.

Cet article prend appui sur l'expérience d'un projet de recherche interdisciplinaire (sciences sociales, agronomie, pihydrologie) et comparatif ${ }^{1}$, réalisé entre 2010 et 2014 sur quatre terrains (Fig. 1) touchés par diverses formes de dégradation de la qualité de l'eau en relation avec les activités agricoles: l'impluvium de Vittel-Contrexéville dans les Vosges, la baie de Douarnenez dans le Finistère, le captage de Harol également dans les Vosges et le lac de la Sorme en Saône-et-Loire. Ces situations se distinguent par l'origine (eau de surface ou eau souterraine) et l'usage de l'eau concernée (eau potable ou eau minérale, eaux littorales, etc.), par les formes d'agriculture pratiquées (élevage hors-sol ou extensif, grandes cultures, etc.) et leurs impacts, mais aussi par le type d'acteurs représentés dans les dispositifs partenariaux de gestion de l'eau, ainsi que par le caractère public ou privé du pilotage (Barataud et al., 2013).

L'analyse qui suit est le fruit d'une démarche réflexive spécifique, menée par quatre chercheuses en sciences sociales issues de disciplines différentes (géographie, anthropologie sociale, sociologie), mais ayant en commun une pratique de la recherche interdisciplinaire autour des questions environnementales. Dans le cadre du projet AGEPEAU, nous avons conduit nos recherches selon une démarche méthodologique commune, inductive, de terrain, basée sur l'entretien semi-directif ou libre, l'observation de situations de réunion, le dépouillement de documentation scientifique et technique et d'archives. Dans le cadre de l'animation scientifique du projet, nous avons réalisé des visites croisées de terrain (Barataud et al., 2013) impliquant l'ensemble des chercheurs. Pour nourrir la démarche réflexive, nous avons aménagé des temps spécifiques entre les chercheuses en sciences sociales, coauteures de cet article. Ensemble, nous avons ainsi construit une grille d'analyse de la diversité des conditions et des formes de notre participation aux dispositifs de gestion de l'eau. Cette grille est fondée sur la chronologie des faits reconstitués a posteriori; elle comprend donc: l'explicitation de l'origine de la recherche dans son contexte local et institutionnel, la caractérisation des dynamiques d'interaction entre parties prenantes, ainsi que des évolutions dans la formulation des problèmes à traiter avec l'irruption de nouveaux sujets et objets. Suivant une démarche de sociologie de la traduction (Callon, 1986), nous avons pris en compte dans cette description à la fois les acteurs et les actants (eau, algues, artefacts de mesure), en considérant que les rôles voire les identités des uns et des autres changent au cours de la recherche (Sedooka et al., 2015). Sur la base de ces matériaux, nous avons débattu de la nature des échanges que nous avons eus avec nos collègues chercheurs en sciences de l'environnement et de l'influence de notre participation sur les relations entre les

\footnotetext{
${ }^{1}$ Il s'agit du projet l'agriculture à l'épreuve des politiques de l'eau (AGEPEAU), conduit sous l'égide du programme «Eaux et territoires » du ministère de l'Écologie, du Développement durable et de l'Énergie.
} 


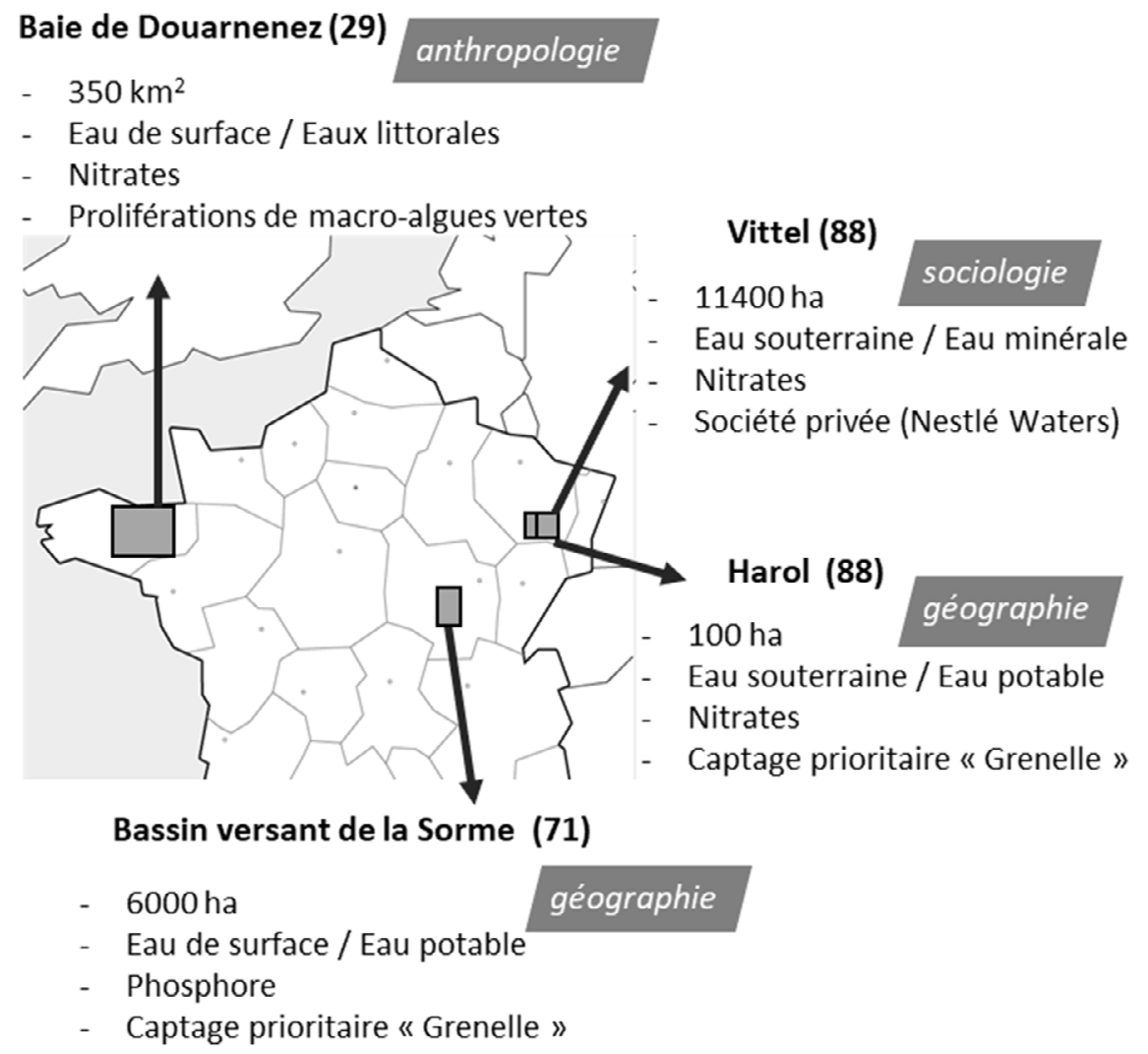

Fig. 1. Des chercheuses engagées sur des terrains.

Source: auteurs.

acteurs d'une part, sur la construction et la mobilisation des connaissances produites d'autre part. Cet article venant deux ans après la fin effective du projet, nous avons également pu observer les conséquences à moyen terme de nos contributions respectives. Les interactions entre disciplines et avec les acteurs peuvent, du fait de ce recul, être saisies à travers une unité de temps, celle de la durée du projet, mais aussi être reliées à une lecture longitudinale d'un terrain où se sont succédé différentes recherches.

Les quatre cas d'étude diffèrent à la fois quant à la nature des problèmes de gestion de l'eau qui s'y posent, et quant à la manière dont les chercheuses en sciences sociales s'y sont impliquées. Leur comparaison nous permet de dégager des enseignements décrivant la variété des cas selon trois ressorts fondamentaux: (i) l'arrivée sur le terrain de chercheuses en sciences sociales en réponse à une demande ou comme proposition heuristique; (ii) la manière dont les sciences sociales négocient leur place; (iii) les rapports qu'elles entretiennent avec l'intervention. Après une première partie reprécisant le cadre théorique dans lequel nous situons cette analyse nous abordons successivement ces trois points à partir d'un regard croisé sur les cas étudiés.

\section{Entre impératifs participatif et scientifique, quel rôle pour les sciences sociales?}

\section{L’impératif participatif}

À partir des années 1960, la participation du public, en prenant en compte des valeurs exprimées dans la société et en corrigeant les logiques administratives et économiques, apparaît comme une réponse au «mécontentement environnemental» (Barbier et Larrue, 2011). Elle alimente dans les années qui suivent la quête d'une «démocratie environnementale» dans laquelle les citoyens et les acteurs associatifs prennent davantage de place dans les processus de décision. Cette injonction à la participation des différentes parties prenantes se retrouve dans un certain nombre de textes réglementaires relatifs à la gestion de l'eau. Elle acquiert progressivement le statut de norme de l'action publique environnementale dans les années 1990; mais les promesses de la participation en termes d'émancipation et de transformation sociales n'ont pas toutes été tenues. Les recherches en sciences sociales contribuent à ce désenchantement 
quand elles montrent qu'en la matière, «bien peu de ce qui est attendu se produit» (Barbier et Larrue, 2011). Elles pointent un risque d'institutionnalisation de la participation, au service d'une acceptabilité sociale des projets qui vise à éviter les conflits et à légitimer l'action publique ou privée, ce cadrage normatif ayant finalement pu limiter la concertation (Pestre, 2011). Les cas que nous étudions ici correspondent justement à des formes de participation institutionnalisée, pilotées par les services de l'État pour les aires d'alimentation de captage (cas de Harol et de la Sorme) et le plan gouvernemental de lutte contre les algues vertes (cas de Douarnenez), ou sous le contrôle d'un acteur privé dans le cas de Vittel.

\section{L'impératif scientifique}

Les quatre cas étudiés ont également en commun une « demande de science », dans le sens d'un appel à la fois à la production de connaissances et à la présence physique des chercheurs (Steyaert, 2006), exprimée par tout ou partie des porteurs d'enjeux. Ces cas apparaissent donc comme exemplaires pour aborder la place des scientifiques dans des dispositifs de gestion de l'eau. Cette place n'est pas neutre. La science est aussi là pour aider à justifier, légitimer et appuyer la décision. Les collègues ou les acteurs réunis attendent parfois des sciences sociales qu'elles prennent en charge le rôle de traducteur, de tiers, d'animateur, de passeur pour rapprocher des acteurs sociaux ou créer des ponts entre des lieux (arènes, institutions, réseaux), ou encore qu'elles servent à légitimer les dispositifs participatifs et les stratégies des décideurs (Barbier et Larrue, 2011; Blondiaux et Fourniau, 2011). Les scientifiques produisent des discours et des argumentaires sur une réalité qu'ils contribuent à transformer (Riaux, 2013a). Ce dernier rôle est souvent peu explicite, que ce soit pour les chercheurs en sciences biotechniques ou sociales. Car «ce que les sciences sociales font à la participation et ce que la participation fait aux sciences sociales » est un sujet qui demande une démarche réflexive (Blondiaux et Fourniau, 2011). La réflexivité, presque inévitable pour les chercheurs impliqués de fait dans des activités d'intermédiation, acquiert alors, lorsqu'il s'agit de chercheurs en sciences sociales, un caractère structurel (Steyaert et al., 2016). Elle constitue en effet à la fois une condition pour la description des dynamiques sociales et politiques engagées sur le terrain et une dimension de leur expérience vécue, dans un contexte où la place des sciences sociales dans ces dispositifs n'est ni acquise, ni stable et doit être construite et négociée chemin faisant.

Ce double appel à la participation et à l'entrée des sciences dans les dispositifs débouche quasi systématiquement sur un appel à l'interdisciplinarité (Hubert et al., 2013) ou à la création d'une «superdiscipline» de l'environnement avec une meilleure pertinence scientifique et sociétale, c'est-à-dire en capacité de résoudre les problèmes (Leroy, 2004). Mais la demande de science formulée par les acteurs des territoires concernés reste principalement adressée aux sciences hydrologiques et agronomiques, pour mieux connaître l'importance et l'itinéraire des flux de polluants (dans nos cas, l'azote et le phosphore) ou les pratiques agricoles alimentant ces flux. Les sciences sociales sont souvent maintenues à la marge par les interlocuteurs de terrain, comme si elles devaient fournir une connaissance accessoire dans la résolution du problème. Dans les cas étudiés, experts et chercheurs en sciences biotechniques et chercheurs en sciences sociales ont des domaines d'application communs (l'eau à l'échelle de bassins versants), mais ni cette communauté, ni la proximité interpersonnelle (Riaux, 2013a) ne garantissent le dialogue interdisciplinaire, car persistent les méconnaissances entre les métiers et une absence de langage commun.

\section{S'inviter dans la gestion de l'eau : quatre situations de recherche}

Nos terrains de recherche se sont construits à l'interface entre une demande plus ou moins clairement formulée de la part des acteurs locaux, et un désir des chercheuses de travailler sur la thématique des relations entre agriculture et gestion de l'eau. Dans cette partie, nous revenons sur la formulation initiale du "problème » de qualité de l'eau sur chacun de ces terrains et précisons les conditions d'entrée des chercheuses sur ceux-ci.

Le dispositif de protection de l'eau mis en place sur l'impluvium de Vittel-Contrexéville par l'entreprise Nestlé à partir des années 1990 vise à maîtriser les risques de pollution par les nitrates des sources d'eau minérale, qui sont liés aux activités agricoles de ce territoire de polyculture-élevage laitier. Des contrats ont ainsi été passés entre des agriculteurs volontaires et une filiale de Nestlé, Agrivair, reposant sur le respect d'un cahier des charges de bonnes pratiques agricoles dont le contenu a été élaboré dans le cadre d'une recherche-intervention (Deffontaines et al., 1993; Barbier et al., 1996; Deffontaines et Brossier, 1997). Or ce dispositif de protection de l'eau, souvent présenté comme exemplaire dans la sphère scientifique et politique (Barraqué et Viavattene, 2009; Hernandez et Benoît, 2011), fait l'objet de critiques de la part de certains acteurs du monde agricole local, qui dénoncent notamment des différences de traitement des éleveurs par Agrivair. C'est ce constat qui a amené la sociologue à revisiter l'histoire de la construction du dispositif de protection de l'eau.

En baie de Douarnenez (Finistère), le programme de recherche débute en 2010 alors que le premier plan gouvernemental de lutte contre les algues vertes est en 
cours de lancement. La baie de Douarnenez est, depuis la fin des années 1970, l'un des principaux sites d'échouage massif d'algues vertes en Bretagne. Le développement des marées vertes est lié à l'importance des flux d'azote au niveau des exutoires des bassins versants, ce que de nombreux travaux produits notamment sous l'égide de l'Ifremer et de l'Inra ont mis en évidence au cours des années 1980 et 1990. La succession de programmes régionaux de lutte contre les algues, auxquels étaient adossés des programmes de recherche, et la mise en œuvre du droit communautaire visant à limiter les apports d'azote sur les parcelles agricoles depuis les années 1990, peinent encore à les faire régresser. Ces politiques, dans un contexte de crise du secteur agricole, sont à l'origine de critiques locales et de résistances de la part des éleveurs, qui pratiquent majoritairement l'élevage hors-sol de porcs, de volaille et de vaches laitières. C'est donc dans un contexte d'inquiétude et de mobilisation des gestionnaires et des porteurs d'enjeux, au sein des instances locales de gouvernance locale de la qualité de l'eau mais également en marge de celles-ci, que l'enquête conduite par une socio-anthropologue prend place ${ }^{2}$, grâce à un soutien des institutions en charge de la qualité de l'eau et une bienveillance des élus locaux, qu'un déplacement du regard sur une question socialement vive intéresse. Ce soutien est toutefois teinté de méfiance et de scepticisme chez certains gestionnaires. Ces réticences sont principalement liées à la densité des controverses locales et régionales sur les marées vertes, qui ont pour effet de renforcer à la fois la demande d'objectivation des processus écologiques en cause, apparaissant comme une priorité en matière de recherche, et le besoin de sécurité des acteurs publics face à des prises de position de chercheurs qui pourraient fragiliser la production tant recherchée d'un consensus.

Harol, petite commune (600 habitants) de l'ouest vosgien, est approvisionnée en eau potable par un captage situé sur le territoire communal, géré en régie communale directe. Ce captage est alimenté par un bassin d'une centaine d'hectares agricoles avec une activité de polyculture-élevage bovin traditionnelle pour le secteur, mais qui inclue une variabilité marquée selon les logiques d'intensification, de mécanisation, d'agrandissement ou d'écologisation à l'œuvre. Le cas de Harol est marqué par la découverte inattendue d'un problème de pollution par les nitrates entraînant, en 2009, le classement du captage en captage prioritaire Grenelle, ce

\footnotetext{
${ }^{2}$ La recherche menée dans AGEPEAU s'inscrit dans le cadre d'un doctorat sous la double tutelle du Muséum national d'histoire naturelle et de l'Inra, et fait l'objet d'un financement partenarial impliquant l'ensemble de la chaîne des acteurs publics concernés par la gestion de la qualité de l'eau en Bretagne, répartissant de fait la charge financière, la responsabilité (et le risque éventuel!) entre chacun d'eux.
}

qui implique pour les acteurs locaux l'élaboration d'un plan d'action selon un processus normé et phasé (Barataud et al., 2014). C'est dans ce contexte que la chercheuse, qui avait pris contact avec la chambre d'agriculture pour identifier un terrain sur lequel conduire une démarche d'accompagnement des acteurs locaux, se voit proposer d'être associée à la procédure Grenelle sur Harol avec l'idée que la démarche qu'elle propose pourra peut-être s'y greffer. Mais les multiples acteurs (institutionnels, professionnels, experts) intervenant sur le secteur se trouvent rapidement confrontés à des incompréhensions et controverses (que ce soit sur le fonctionnement hydrogéologique et la taille de l'aire, sur une vulnérabilité particulière des sols, ou encore sur les objectifs à atteindre) qui tendent à prendre le pas sur la proposition initiale de la chercheuse.

En 2009, la procédure Grenelle inclut dans la liste des captages prioritaires le lac de la Sorme, principale ressource en eau potable de l'agglomération du CreusotMontceau-Les-Mines. L'important programme de mise aux normes des exploitations d'élevage déployé de 1995 à 2001 n'a pas permis de juguler l'eutrophisation ${ }^{3}$ du lac (Petit, 2015). Les expertises des consultants pointent des apports excessifs en phosphore du bassin versant et donc de l'élevage, pourtant fondé sur le pâturage extensif de troupeaux de vaches allaitantes. La chambre d'agriculture de Saône-et-Loire sollicite le laboratoire Carrtel de l'Inra, spécialiste de l'écologie des lacs, pour mieux comprendre les flux de phosphore. Elle mise sur le crédit de l'Inra pour que le diagnostic soit légitime aux yeux des agriculteurs, car ceux-ci craignent de nouvelles règles interdisant l'accès des bovins aux ruisseaux. Ils rappellent les origines artificielles du lac, en cause, selon eux, dans la dégradation de la qualité de l'eau. La chambre d'agriculture sollicite alors un éclairage par les sciences sociales, car les gestionnaires du lac craignent un refus de plan d'action par les agriculteurs. S'initie ainsi une collaboration entre la communauté urbaine du Creusot-Montceau et deux chercheurs de l'Inra (l'un avec une double compétence en hydrologie et en agronomie, l'autre avec une compétence en géographie sociale), auxquels l'appartenance institutionnelle donne de la légitimité.

C'est dans les cas de la Sorme et d'Harol que la demande a été la plus explicite et directement liée aux politiques publiques. L'obligation pour la collectivité d'engager une démarche spécifique a conduit sur la Sorme à des collaborations avec l'Inra, la chambre d'agriculture jouant un rôle d'intermédiaire. Sur Harol, la petite collectivité, confrontée à une situation inédite à laquelle elle n'était pas préparée, a répondu favora-

\footnotetext{
${ }^{3}$ L'eutrophisation peut être définie comme une altération multi-symptomatique des milieux aquatiques liée à des apports excessifs en nutriments.
} 
blement à la sollicitation (par l'entremise de la chambre d'agriculture) de la chercheuse en quête d'un terrain. Dans ces deux cas, les collectivités mises en demeure d'agir étaient en recherche d'appuis méthodologiques et de réponses techniques sur les mesures à mettre en œuvre. Elles ont alors ouvert la porte aux chercheurs. À Vittel et en baie de Douarnenez, c'est une connaissance préalable du territoire qui a fait percevoir aux chercheuses que les recherches antérieures n'avaient pas permis de saisir tous les enjeux sociaux associés à la protection de l'eau, qu'il restait donc des zones d'ombre à explorer. Les chercheuses ne se sont alors pas associées à un acteur précis comme cela a été le cas avec la chambre d'agriculture sur les deux autres terrains, préférant circuler librement parmi l'ensemble des acteurs de ces terrains.

Sur tous nos terrains, de multiples connaissances ont été produites ou convoquées : le savoir construit par des cabinets d'expertise ${ }^{4}$ (en particulier en hydrogéologie); la connaissance ordinaire du territoire et des pratiques agricoles pouvant être portée par la chambre d'agriculture ou par certains élus issus du milieu agricole; parfois des compétences d'ingénierie des collectivités locales. À ces savoirs, sur trois des cas étudiés se sont ajoutés des apports scientifiques, essentiellement issus des sciences agronomiques ou hydrogéologiques. Comme nous l'avons indiqué, la baie de Douarnenez a fait l'objet d'une multiplicité d'expertises. Sur la Sorme, il s'agissait uniquement d'expertises hydrologiques. Sur Vittel, il s'agissait de travaux assez anciens, mais qui continuent de façonner l'image que ce territoire offre à l'extérieur du département. Des recherches en sciences sociales ont également été menées, centrées sur la construction du dispositif de protection de l'eau et le rôle joué par la recherche (Barbier, 2008). Par opposition, il n'existait aucun travail de recherche ni d'expertise sur Harol avant son classement en captage Grenelle.

Si l'analyse empirique des discours et des pratiques des protagonistes n'est pas perçue d'emblée comme un savoir spécialisé contribuant à la connaissance des territoires hydrologiques, il n'en existe pas moins une attente vis-àvis des sciences sociales. Mais celle-ci ne semble pas du même ordre: le chercheur est alors davantage perçu comme ayant une fonction d'aide à la prise de recul et de médiation. Ce sont les évolutions dans ces attentes et la manière dont les sciences sociales construisent leur place et influent à la fois sur les dynamiques et la formulation des questions que nous allons exposer maintenant.

\footnotetext{
${ }^{4}$ Nous retenons comme définition de l'expertise, l'« expression d'une connaissance ou fourniture d'une synthèse des connaissances disponibles sur un sujet donné» (Coreau et al., 2013), formulée en réponse à la demande de ceux qui ont une décision à prendre. Roqueplo (1997) ajoute «et en sachant que cette réponse est destinée à être intégrée au processus de décision».
}

\section{Les sciences sociales font leurs preuves}

\section{Les sciences sociales en posture de tiers}

Totalement absentes (dans le cas d'Agrivair sur Vittel) ou tardives (sur la Sorme, en aval du diagnostic), les attentes vis-à-vis des sciences sociales nous ont paru peu formalisées en termes de connaissances à produire. Elles restent, sinon marginales, du moins très spécifiques. Elles se manifestent essentiellement lors de deux temps particuliers: d'une part, l'entretien avec les acteurs du terrain et, d'autre part, la restitution promise des travaux. L'entretien apparaît en effet pour les acteurs comme une occasion de pouvoir témoigner, rétablir une (sa) vérité et être (enfin) entendu, dans l'espoir aussi que le message sera transmis. Ainsi, en baie de Douarnenez, la conscience chez les acteurs de la singularité et de l'épaisseur de leur expérience vécue des relations entre sciences, politique et société a fait que la grande majorité d'entre eux ont jugé d'emblée intéressant d'en témoigner auprès d'un tiers perçu comme extérieur. Les motivations des personnes interviewées à la prise de parole devant l'enquêtrice étaient souvent clairement formulées: rendre justice à la spécificité du territoire dans le cadre de plans de lutte pensés de façon assez générique aux échelles nationale et régionale pour certains élus et certains responsables agricoles; documenter les freins «psychologiques» au changement pour certains gestionnaires; réhabiliter une image perçue comme largement malmenée par des accusations injustes pour de nombreux agriculteurs; déconstruire les discours des «marchands de doute» niant l'origine agricole des nitrates favorisant les proliférations d'algues et dénoncer l'emprise des industries agroalimentaires qui les emploient pour certains militants environnementalistes, rejoints en cela par une partie des chercheurs en sciences biophysiques que la question des marées vertes avait occupés dans les décennies précédentes.

Après l'entretien, la restitution apparaît comme une autre étape du travail qui intéresse potentiellement les acteurs. Ainsi sur Vittel, certains acteurs enquêtés, principalement des agents de la chambre d'agriculture, se sont montrés très intéressés par les résultats de l'étude. Ces derniers avaient été en effet exclus de la construction du dispositif de protection de l'eau, ils n'avaient pas été associés à la définition du cahier des charges de bonnes pratiques agricoles et entretenaient des relations difficiles avec le directeur d'Agrivair. Surtout, ils avaient observé que ce dernier avait négocié de manière individuelle les contrats avec les agriculteurs, ce qui était source d'inégalités, et ils étaient désireux d'en savoir plus. Les résultats du travail sociologique sont alors attendus pour dévoiler un pan de l'histoire du territoire qu'il est difficile d'aborder, ou pour faire entendre des récits minoritaires. 
Les chercheurs en sciences sociales sont ainsi à la « merci du moment» (Becker, 2002) et leurs interlocuteurs ne sont pas des acteurs passifs, dociles à l'enquête, impuissants. Au contraire, ces derniers peuvent rechercher par le biais de l'enquêteur à faire valoir un point de vue faisant de l'enquête un moment de négociation au cours duquel les positions de l'enquêté et de l'enquêteur se transforment (Becker, 2016).

\section{Contribution des sciences sociales à la compréhension des situations}

Sur nos différents terrains, c'est la capacité à montrer une autre façon de "connaître» le territoire lors des réunions, ou ex post (dans des phases de restitution écrite par exemple), et à permettre que soit réfléchie la place que le collectif souhaite donner aux différents systèmes de sens des acteurs qui a ensuite intéressé nos interlocuteurs. Par exemple, sur Harol, la relation de confiance instaurée avec les agriculteurs et le maire a fait que la chercheuse a été sollicitée pour accompagner le maire dans la présentation de son cas lors d'une journée départementale sur les captages à la chambre d'agriculture. Le savoir-faire qui lui a été reconnu, c'est une capacité à opérer une forme de « traduction scientifique » (Beuret, 2006), c'est-à-dire à « traduire le réel pour le rendre intelligible par tous ». La situation est alors appréhendée sous un nouveau jour, par la mise en visibilité d'acteurs, d'expériences et de paroles silencieuses et par la capacité à en faire le récit.

De même, l'approche compréhensive sur la Sorme a permis de reconstituer une « histoire» du lac aux côtés du volet hydrologique. Pour les agriculteurs, les mauvaises conditions de création du lac sont une des causes de la mauvaise qualité de l'eau. Cet argument pourrait sembler défensif face à de nouvelles mesures perçues comme des contraintes supplémentaires dans un contexte économique difficile. Pourtant, notre recherche a montré qu'il s'ancre plus profondément dans la représentation du lac comme un espace créé par l'homme devant être entretenu de façon analogue aux mares régulièrement curées par les agriculteurs pour enlever la vase. Cette représentation se heurte à une autre représentation du lac et du bassin versant, celle d'un écosystème. Une des conclusions du travail conduit par la géographe sur ce terrain est que la dissonance entre les deux visions du lac et de son bassin versant doit se résorber pour que les agriculteurs s'engagent dans des plans d'action (Petit, 2015). Sur l'impluvium de Vittel-Contrexéville, l'enquête conduite par la sociologue a permis de mettre en évidence le rôle central que les questions foncières ont joué dans l'acceptation par les agriculteurs des contrats environnementaux : le directeur d'Agrivair est en effet parvenu à acquérir d'importantes surfaces agricoles sur ce territoire, ensuite mises à disposition gratuitement des seuls agriculteurs signataires. Ainsi, la recherche a permis d'examiner un pan du dispositif de protection de l'eau mis en place à Vittel, jusque-là resté dans l'ombre (Hellec, 2015). Cela a également permis d'en relativiser le caractère exemplaire, dès lors que les moyens mis en œuvre pour l'acquisition des terres agricoles sont difficilement mobilisables par des acteurs publics (Barataud et Hellec, 2015).

Sur nos terrains, nous avons constaté que les débats d'experts spécialisés laissent peu de place à l'« expertise d'usage » ou aux «savoirs d'usage» (Barbier et Larrue, 2011; Sintomer, 2008). En particulier, les savoirs des agriculteurs, en termes de dynamique de l'eau et de la végétation, de phénomènes d'érosion sur les bassins versants, ou encore de différenciation des types de sols, ne sont généralement pas mobilisés comme des savoirs pertinents dans l'élaboration des diagnostics (Petit et Barataud, 2015). Ainsi, au prisme des savoirs, la participation de tous n'est pas acquise et le «continuum d'expertise du profane total au scientifique le plus spécialisé » (De Carvalho, 2013) n'est pas reconnu ou mobilisé de manière constante. L'attention aux savoirs et à leur inégale mobilisation dans la gouvernance des pollutions agricoles révèle aussi leur haut degré de politisation: les acteurs locaux de la qualité de l'eau ont développé au fil du temps de véritables politiques de la connaissance et se sont outillés pour les défendre (Levain et al., 2015; Levain, 2017). En effet, les indicateurs ne sont pas que des mesures objectives, ils résultent de bricolages combinant des savoirs empiriques et scientifiques, et ce sont dans ces interstices que se nichent des intérêts et des jeux de pouvoirs (Riaux, 2013b).

Grâce au travail des sciences sociales, les récits des territoires passent du statut d'arrière-plan connu de quelques acteurs disposant d'un fort ancrage territorial à une dimension à part entière du problème à traiter. La définition de ce problème inclut alors les conditions effectives de la mobilisation, les asymétries affectant les relations entre acteurs, ainsi que l'évolution des conditions de légitimité des activités agricoles sur le territoire. Ce faisant, le chercheur en sciences sociales participe au développement des processus d'intermédiation qui désectorisent les politiques de l'eau, et sont susceptibles d'améliorer, parce qu'ils les diversifient, les formes d'interactions sociales et les apprentissages qui accompagnent ces dernières (Billaud et al., 2013).

\section{Une place qui se construit chemin faisant}

Nos travaux ont produit différentes connaissances sur ces cas. Les principaux résultats ont concerné la construction collective de nouveaux dispositifs territoriaux pour la gestion des rapports entre agriculture et qualité de l'eau, l'évolution locale de l'agriculture et du métier d'agriculteur (Hellec, 2015), le rôle de différents savoirs dans l'élaboration de solutions de gestion (Petit et 
Barataud, 2015), les épreuves indissociablement cognitives et politiques que traversent les acteurs impliqués dans la gestion locale de la qualité de l'eau (Barataud et al., 2013 ; Levain, 2013 ; Levain, 2014 ; Levain, 2017).

Les jeux d'alliance ou de concurrence qui se nouent autour de la production/réception des savoirs ont joué sur la place et le statut qui nous ont été accordés au fil du temps sur nos terrains d'enquête. Par exemple, sur Harol, la chercheuse a été d'emblée associée au comité de pilotage et à la démarche Grenelle par la chambre d'agriculture, avec laquelle elle est donc apparue en tandem. Disposant par ailleurs d'une double compétence en sciences sociales et en sciences de l'environnement, elle s'est ensuite systématiquement trouvée ramenée en situation d'expertise technique (acquérant par là même sa légitimité à suivre et à participer à la démarche) et, parfois, quasiment de contrepouvoir face à un bureau d'études en hydrogéologie dont les compétences étaient régulièrement remises en cause en comité de pilotage.

Qu'ils soient intégrés à un collectif interdisciplinaire ou disposent, comme dans le cas de Harol, d'une double compétence en sciences sociales et en sciences de l'environnement, les chercheurs en sciences sociales sont dans une position instable et ambiguë. Ils peuvent vouloir la cultiver, dans l'objectif de conserver l'accès au terrain, de bénéficier d'une légitimité supplémentaire, et/ou de maintenir leur intégration dans un collectif de recherche dans lequel ils sont minoritaires. Lorsqu'ils sont seuls à intervenir, la marginalité et la faiblesse des attentes initiales exprimées sont une ressource pour conduire des enquêtes indépendantes en contexte conflictuel. Leur contribution s'apparente alors à une expertise qui ne propose ni ne conçoit de solution, mais qui permet d'objectiver la controverse et a, en cela, pour fonction d'aider à résoudre le conflit (Vandevelde, 2013). Le risque est alors cependant de ne pas parvenir à opérer la liaison, toujours fragile, avec l'action publique alors même que, de fait, l'action publique est un des éléments constitutifs du terrain auquel leur appartenance à un institut de recherche finalisée et leurs choix individuels les rendent sensibles.

\section{Les sciences sociales en recherche- intervention: surprises et ramifications}

\section{La mobilisation des connaissances: des trajectoires inattendues}

Sur les territoires, la demande en connaissances hydrologiques et agronomiques place d'emblée les experts et les scientifiques de ces disciplines dans une posture d'autorité, la légitimité des savoirs contribuant à une légitimité sociale (Pestre, 2011). Pourtant, ces recherches biophysiques peuvent contribuer à dévoiler de nouvelles incertitudes, rendant ainsi le processus de gestion et la prise de décision plus complexes et provoquant une possible déstabilisation des décideurs (Callon et al., 2001). Dans ce cas, le recours à des spécialistes n'atteint pas l'objectif escompté par les décideurs et/ou gestionnaires, à savoir fonder et légitimer leurs décisions (Granjou et al., 2010). Les incertitudes qui laissent ouvertes des controverses (Marc et Blanc, 2013) dessinent un espace de travail et d'observation aux sciences sociales, car dans ce cas les rapports de force et de légitimité de savoirs entre acteurs restent au cœur des décisions. L'offre de connaissances des sciences sociales n'est pas immédiatement perçue comme pertinente par les autres disciplines et pour la décision. Elle peut même être redoutée, quand elle opère un dévoilement des rapports de force entre acteurs ou des conséquences des décisions prises. Donner une caution scientifique aux dispositifs mis en œuvre, ou s'adjoindre un regard extérieur venant compléter leur propre analyse, sont les deux motivations des gestionnaires pour solliciter (ou accepter) les apports des sciences sociales. Toutefois, l'absence de demande ou le flou l'entourant a permis aux chercheuses de se dégager des injonctions usuelles que les sciences biotechniques adressent aux sciences sociales autour de «l' acceptabilité » de nouvelles pratiques de la part des agriculteurs (Compagnone, 2015).

Dans les quatre cas, les connaissances que nous avons produites n'ont pas immédiatement conforté l'opérationnalité des politiques de gestion des pollutions diffuses, ou la légitimité de leurs porteurs. Aucune des expériences dont nous avons rendu compte ne s'est traduite, au moins à court terme, par une intégration des recherches en sciences humaines et sociales au «pool» des connaissances jugées indispensables pour décrire et prendre en charge une situation problématique: les «sciences» restent globalement entendues par nos interlocuteurs comme visant l'objectivation de processus complexes qui sont avant tout de nature biologique et physique. La dimension sociale de cette complexité, que les sciences sociales contribuent à éclairer, reste appréhendée comme un élément contextuel d'une part, une question politique et opérationnelle d'autre part.

Il en va différemment lorsque l'on raisonne sur des échelles de temps et d'espace plus étendues. Pour identifier la façon dont les acteurs sociaux se sont saisis des apports des sciences sociales, une perspective élargie est en effet nécessaire. Élargissement, d'abord, vers d'autres niveaux d'organisation et de gestion: en Bretagne, l'organisation de l'expertise scientifique a été considérablement revue à l'occasion de la rédaction du deuxième plan régional de lutte contre les algues vertes (2017-2022), en positionnant l'ensemble des chercheurs plutôt dans une perspective d'accompagnement que d'évaluation. Les chercheurs en sciences sociales, initialement très peu représentés, y occupent désormais une place comparable à 
celle des chercheurs en sciences biophysiques. Élargissement, ensuite, vers d'autres situations et postures de recherche : l'introduction d'un regard en sciences sociales fait que la chercheuse qui a travaillé sur le captage de Harol est désormais sollicitée par l'agence de l'eau Rhin-Meuse, qui lui reconnaît d'emblée un rôle en terme d'apports compréhensifs (sur les réseaux d'acteurs, sur l'inscription spatiale des activités et des liens entre acteurs), pour intervenir dans des configurations comparables. Élargissement, enfin, dans le sens où les interprétations proposées par les chercheuses du projet donnent à ces situations une visibilité externe: la majorité des sollicitations de gestionnaires, d'associations, d'organisations professionnelles dont elles sont aujourd'hui l'objet émanent d'acteurs extérieurs aux territoires étudiés.

Quoi qu'il en soit, la connexion entre la recherche et l'action peut s'avérer délicate : elle demande à être flexible sur le calendrier et à concevoir des actions selon une logique d'adaptation au fur et à mesure de l'acquisition de connaissances. Les dispositifs que nous avons décrits sont pour tous, y compris les chercheurs, des situations d'apprentissage cognitif et social (Barbier et Larrue, 2011). Pour la recherche, c'est une épreuve en termes d'enjeux de pédagogie sur la compréhension de la nonlinéarité des systèmes socioécologiques observés. C'est aussi une situation de transmission de connaissances délicate car les résultats «n'arrangent» pas toujours les agriculteurs quand leurs pratiques sont mises en cause, ou les gestionnaires quand les résultats pointent les effets antagonistes des actions qui pourraient être mises en œuvre. C'est, enfin, une source infinie de remise en question de la qualité et de la pertinence des connaissances produites.

\section{Un engagement qui dépasse le cadre initialement prévu}

Dans tous les cas, la confrontation avec les acteurs locaux s'est révélée tout aussi enrichissante qu'inconfortable, car elle renvoie au rapport que nous, chercheurs, entretenons avec l'action et à notre dépendance vis-à-vis de la dynamique des situations. Nous avons toutes observé que notre implication auprès des acteurs se déployait sur les plans relationnel et temporel bien audelà du cadre initialement prévu.

La production des chercheurs en sciences biophysiques, en particulier la validation empirique des modèles de transfert de nutriments, n'est pas seulement un outil de calibration des processus ou de prévision de tendances évolutives. C'est également un support possible de réflexion et de dialogue, qui peut se trouver en capacité de faire émerger de nouveaux paradigmes ou modes de représentation des systèmes environnementaux. En ce sens, l'intégration dans un cadre de travail interdisci- plinaire a pu constituer un point d'appui considérable sur deux des territoires d'étude, au moins. Cependant, l'émergence de ce travail en interdisciplinarité reste subordonnée à l'existence de travaux et d'interaction dans la longue durée.

Notre implication forte sur ces terrains nous a permis d'accéder à des scènes d'observation privilégiées et a donc nourri directement nos analyses et interprétations. Les interactions avec les différents partenaires, tant dans la forme qu'elles prennent que dans leur contenu, ont également constitué des sources d'information précieuses. Par conséquent, l'engagement du chercheur en sciences sociales sur le terrain nous semble être un moyen d'analyser de manière plus précise, et donc dans toute leur complexité, des problèmes socioenvironnementaux qui, sans cela, restent difficilement lisibles. Sur ces terrains hybrides qu'il faut appréhender, avec nos interlocuteurs, comme des situations problématiques, les conditions de la distinction entre le «dedans» et le «dehors», entre l'engagement et la prise de distance, font l'objet d'une «boucle dynamique» et d'un ajustement constant, qui appellent la réflexivité sur les connaissances produites et leur valeur relative (Müller, 2015). Ainsi, par-delà les cultures disciplinaires et les sensibilités différentes des chercheuses en sciences sociales impliquées dans ces quatre situations, l'engagement auprès des acteurs et les ajustements qui l'accompagnent se traduisent par une évolution des questions de recherche initiales vers des questions sur le statut des connaissances dans les dispositifs participatifs de gestion des problèmes environnementaux complexes : c'est ainsi en identifiant la nature relationnelle et dialogique des savoirs produits par l'ensemble des parties prenantes que nous avons construit, dans la pratique et à partir d'une position marginale, des connaissances interdisciplinaires.

\section{Conclusion}

Les enjeux et les effets de l'engagement des chercheurs dans les démarches interdisciplinaires que nécessitent la compréhension et la prise en charge des problèmes environnementaux complexes sont dépendants des disciplines et des postures scientifiques envisagées. Ainsi, la place de la recherche qualitative en sciences sociales dans les dispositifs de gestion de l'eau n'est jamais totalement acquise, parce qu'elle produit des connaissances essentiellement dialogiques, dont les objets se déplacent et se transforment au fil des enquêtes et des interactions avec les porteurs d'enjeux. La nature de son apport reste ainsi fondamentalement incertaine, dans un contexte où les porteurs d'enjeux attribuent majoritairement à l'implication de la recherche une fonction de réduction de l'incertitude, qu'elle soit institutionnelle ou plus fondamentalement liée aux dynamiques des systèmes étudiés. 
Si l'expérience de chaque chercheur impliqué dans le projet AGEPEAU est singulière, à la fois du fait de la diversité des contextes dans lesquels les chercheurs sont intervenus et des spécialités qu'ils représentent, des éléments de culture commune se sont dégagés de plus en plus clairement au fil du projet : ils cherchaient tous, par leur engagement, à contribuer à la structuration d'un espace et d'une décision publique plus démocratiques. Pour autant, dans les quatre cas présentés dans cet article, il nous semble que l'implication des chercheuses en sciences sociales n'a finalement pas conduit à modifier les rapports de force locaux, ceux-ci s'étant simplement déplacés ou exprimés différemment, ce qui peut cependant être considéré comme un rôle, même modeste, sur le cours de l'action. Et c'est finalement moins par l'intervention directe que par la réorientation de nos questions de recherche et de nos publications vers des problématiques touchant directement les acteurs que nous avons pu partiellement traiter un certain nombre d'enjeux : en nous attachant à restituer la richesse des savoirs d'expérience et en analysant la façon dont ils se trouvaient exclus de l'équipement cognitif des dispositifs d'action publique dans le domaine de l'eau, en interrogeant les enjeux de justice environnementale sous-jacents dans les politiques de lutte contre les pollutions diffuses d'origine agricole, en mettant en évidence les contradictions entre le poids croissant des responsabilités confiées aux acteurs locaux en la matière et la modicité des moyens et des leviers dont ils disposent.

Il nous semble alors que l'autorité des chercheurs en sciences humaines et sociales impliqués dans des projets se construit sur des bases plus complexes et plus contextuelles que celles des chercheurs en sciences biophysiques, et bien souvent en interaction étroite avec ces disciplines scientifiques (Lits, 2013; Grisoni et Némoz, 2013), qui interrogent le positionnement de la sociologie dans des collaborations scientifiques pluridisciplinaires face aux enjeux environnementaux. Les sciences sociales peuvent ainsi parvenir, par la finesse des observations et la dimension incarnée des connaissances qu'elles produisent, à entrer en résonnance avec l'expérience vécue de problèmes environnementaux complexes, comme celui de la gestion des pollutions diffuses d'origine agricole.

\section{Remerciements}

Cette recherche a nécessité l'implication active de nombreux partenaires locaux, parmi lesquels l'établissement public d'aménagement de la baie de Douarnenez, la commune d'Harol, la communauté urbaine du CreusotMontceau-les-Mines, les chambres d'agriculture 88 et 71 , que nous tenons à remercier chaleureusement. Nous remercions également l'ensemble des personnes qui ont accepté de nous accorder un entretien et de partager avec nous leurs réflexions, leurs doutes, leurs connaissances.

\section{Références}

Barataud F., Barbier M., Benoît M., Blouet A., Doidy E., Hellec F., Levain A., Petit S., Trévisan D., 2013. L'agriculture à l'épreuve des politiques de l'eau (projet $A G E P E A U)$. Rapport final, Paris, ministère de l'Écologie, du Développement durable et de l'Énergie, http://isidoredd. documentation.developpement-durable.gouv.fr/documents/ Temis/0081/Temis-0081323/21804_A.pdf.

Barataud F., Durpoix A., Mignolet C., 2014. Broad analysis of French priority catchment areas: a step toward adaption of the Water Framework Directive?, Land Use Policy, 36, $427-$ 440, doi: 10.1016/j.landusepol.2013.09.010.

Barataud F., Hellec F., 2015. L'outil foncier, une solution délicate pour protéger les captages d'eau potable, Économie rurale, 347, 3, 3-20, doi: 10.4000/economieru rale.4634.

Barbier M., 2008. Bottling water, greening farmers: the sociotechnical and managerial construction of a "dispositif" for underground water quality protection, International Journal of Agricultural Resources Governance and Ecology, 7, 1-2, 174-197, doi: 10.1504/IJARGE.2008.016986.

Barbier M., Benoît M., Brossier J., Chia E., Deffontaines J.-P., Gras F., Lemery B., Roux M., 1996. Programme de recherche Agriculture-Environnement-Vittel (AGREV). Rapport de synthèse, Société des Eaux de Vittel, http:// cdi.eau-rhin-meuse.fr/GEIDEFile/fichier.pdf?Archive= 111233593941.

Barbier R., Larrue C., 2011. Démocratie environnementale et territoires : un bilan d'étape, Participations, 1, 1, 67-104, doi: 10.3917/parti.001.0067.

Barraqué B., Viavattene C., 2009. Eau des Villes et Eau des Champs. Vers des accords coopératifs entre services publics et agriculteurs?, Économie rurale, 310, 2, 5-20, doi: 10.4000/economierurale. 708 .

Becker H., 2002. Les ficelles du métier. Comment conduire sa recherche en sciences sociales, Paris, La Découverte.

Becker H., 2016. La bonne focale. De l'utilité des cas particuliers en sciences sociales, Paris, La Découverte.

Beuret J.E., 2006. La conduite de la concertation pour la gestion de l'environnement et le partage des ressources, Paris, L'Harmattan.

Billaud J.-P., Catalon E., Steyaert P., 2013. De l'instrumentation de la gestion de l'eau à sa territorialisation: objets, savoirs, acteurs. Rapport final, Paris, ministère de l'Écologie, du Développement durable et de l'Énergie, https://reseaux.parisnanterre.fr/wp-content/uploads/2013/ 05/Rapport-Final-OSA-Billaud.pdf.

Blondiaux L, Sintomer Y., 2009. L’impératif délibératif, Rue Descartes, 63, 1, 28-38.

Blondiaux L., Fourniau J.-M., 2011. Un bilan des recherches sur la participation du public en démocratie : beaucoup de bruit pour rien?, Participations, 1, 1, 8-35, doi: 10.3917/ parti.001.0008.

Bouleau G., Richard S., 2008. Les lois sur l'eau à la lumière de la directive cadre, Montpellier, AgroParisTech.

Brun A., 2011. La gestion de l'eau par bassin versant en France: un «modèle» en sursis ?, in Brun A., Lasserre F. 
(Eds), Gestion de l'eau-Approche territoriale et institutionnelle, Québec, PUQ, 63-93.

Callon M., 1986. Some elements of a sociology of translation: domestication of the scallops and the fisherman of St Brieuc bay, in Law J. (Ed.), Power, action and belief: a new sociology of knowledge, London, Routledge, 196-232.

Callon M., Lascoumes P., Barthe Y., 2001. Agir dans un monde incertain. Essai sur la démocratie technique, Paris, Le Seuil.

Carvalho (de) L., 2013. Le complexe nucléaire de Sellafield au cœur des controverses : le fait technologique entre experts et profane, VertigO, 13, 2, doi: 10.4000/vertigo.14187.

Compagnone C., 2015. Saisir les pratiques agricoles respectueuses de l'environnement. Quelle posture du sociologue face à l'agronome?, Sciences de la société, 96, 119-135.

Coreau A., Nowak C., Mermet L., 2013. L'expertise pour les politiques nationales de biodiversité en France: quelles stratégies face aux mutations en cours ?, VertigO, 13, 2, doi: 10.4000/vertigo.14040.

Deffontaines J.-P., Benoît M., Brossier J., Chia E., Gras F., Roux M., 1993. Agriculture et qualité des eaux. Diagnostic et propositions pour un périmètre de protection, Versailles, Inra Éditions.

Deffontaines J.-P., Brossier J. (Eds), 1997. Agriculture et qualité de l'eau. L'exemple de Vittel, Versailles, Inra Éditions.

Granjou C., Mauz I., Cosson A., 2010. Le recours aux savoirs dans l'action publique environnementale : un foisonnement expérimental, Sciences de la société, 79, 115-129.

Grisoni A., Némoz S., 2013. La sociologie, discipline experte des controverses environnementales ?, VertigO, 13, 2, doi: 10.4000/vertigo.14157.

Hellec F., 2015. Revenir sur l'exemplarité du cas de Vittel: formes et détours de l'écologisation d'un territoire agricole, VertigO, 15, 1, http://vertigo.revues.org/15912; doi: $10.4000 /$ vertigo. 15912 .

Hernandez S., Benoît M., 2011. Gestion durable de la ressource en eau : l'utilisation du paiement pour service environnemental au service de la protection des captages, Annales des Mines-Responsabilité et environnement, 63, 87-95.

Hubert B., Aubertin C., Billaud J., 2013. Recherches participatives, recherches citoyennes.. une clarification nécessaire, Natures Sciences Sociétés, 21, 1, 1-2, doi: $10.1051 / \mathrm{nss} / 2013078$.

Leroy P., 2004. Sciences environnementales et interdisciplinarité : une réflexion partant des débats aux Pays-Bas, Natures Sciences Sociétés, 12, 3, 274-284, doi: 10.1051/ nss:2004039.

Levain A., 2013. Faire face aux marées vertes, penser les crises du vivant, Ethnographiques.org, 27, www.ethnographi ques.org/2013/Levain.

Levain A., 2014. Pour innover, vivons cachés? Ce que c'est que d'être pilote, in Gascuel C., Ruiz L., Vertès F. (Eds), Comment réconcilier agriculture et littoral? Pour une agroécologie des territoires, Versailles, Quae, 103-135.

Levain A., 2017. Une épreuve médiatique? Les éleveurs bretons et les marées vertes, Études rurales, 198, 171-194, https://www.cairn.info/revue-etudes-rurales-2016-2-page171.htm? contenu=resume.

Levain A., Vertès F., Ruiz L., Delaby L., Gascuel-Odoux C., Barbier M., 2015. "I am an intensive guy": the possibility and conditions of a reconciliation through the ecological intensification framework, Environmental Management, 56, 5, 1184-1198, doi: 10.1007/s00267-015-0548-3.

Lits G., 2013. Analyse du rôle des chercheurs en sciences sociales dans la gestion des déchets radioactifs, VertigO, 13, 2, http:// vertigo.revues.org/14207; doi: 10.4000/vertigo.14207.

Marc D., Blanc F., 2013. Comment légitimer l'accession au statut d'expert pour limiter les controverses : étude de cas en biodiversité, VertigO, 13, 2, doi: 10.4000/vertigo.14033.

Müller A., 2015. Altérités et affinités ethnographiques : réflexions autour du proche, du lointain, du dedans et $\mathrm{du}$ dehors, SociologieS, http://sociologies.revues.org/4906.

Narcy J.-B., 2013. Regards des sciences sociales sur la mise en ouvre des politiques de l'eau, Onema, www.gesteau.fr/ sites/default/files/dim_so.pdf.

Parris K., 2011. Impact of agriculture on water pollution in OECD countries: recent trends and future prospects, International Journal of Water Resources Development, 27, 1, 33-52, doi: 10.1080/07900627.2010.531898.

Pestre D., 2011. Des sciences, des techniques et de l'ordre démocratique et participatif, Participations, 1, 1, 210-238, doi: 10.3917/parti.001.0210.

Petit S., 2015. Au fond de l'eau : histoires sociales et représentations environnementales d'un bassin versant agricole, Territoire en mouvement, 25-26, http://tem. revues.org/2757.

Petit S., Barataud F., 2015. L'eau, source de savoirs. Analyse de situations d'expertise dans des bassins versants agricoles, VertigO, 15, 1, doi: 10.4000/vertigo.15938.

Raadgever G.T., Dieperink C., Driessen P.P.J., Smit A.A.H., Van Rijswick H.F.M.W., 2011. Uncertainty management strategies: lessons from the regional implementation of the Water Framework Directive in the Netherlands, Environmental Science and Policy, 14, 1, 64-75, doi: 10.1016/j. envsci.2010.11.001.

Riaux J., 2013a. Engager la construction d'un regard sociohydrologique: des archives catalyseurs de l'interdisciplinarité, Natures Sciences Sociétés, 21, 1, 15-23, doi: $10.1051 / \mathrm{nss} / 2013061$.

Riaux J., 2013b. Ethnographie d'un dispositif de gestion publique des eaux : à propos des paradoxes de la « mesure» hydrologique, Journal des anthropologues, 132-133, 361-381, doi: 10.4000/jda.5037.

Roqueplo P., 1997. Entre savoir et décision, l'expertise scientifique, Versailles, Quae.

Sedooka A., Steffen G., Paulsen T., Darbellay F., 2015. Paradoxe identitaire et interdisciplinarité : un regard sur les identités disciplinaires des chercheurs, Natures Sciences Sociétés, 23, 4, 367-377, doi: 10.1051/nss/2015056.

Sintomer Y., 2008. Du savoir d'usage au métier de citoyen ?, Raisons politiques, 31, 3, 115-133, doi: 10.3917/ rai.031.0115. 
Steyaert P., 2006. Dispositifs d'action collective : un concept pour comprendre la gestion concertée de l'eau à l'échelle de bassins versants, in Mérot P. (Ed.), Qualité de l'eau en milieu rural, savoirs et pratiques dans les bassins versants, Versailles, Quae, 255-266.

Steyaert P., Ollivier G., 2007. The European Water Framework Directive: how ecological assumptions frame technical and social change, Ecology and Society, 12, 1, 25, www. ecologyandsociety.org/vol12/iss1/art25.
Steyaert P., Barbier M., Cerf M., Levain A., Loconto A.M., 2016. Role of intermediation in the management of complex sociotechnical transitions, AgroEcological Transitions, Wageningen University Research (version préprint disponible sur Hal-SHS, https://hal.archives-ouver tes.fr/hal-01470892/document).

Vandevelde J.-C., 2013. L'outarde et le TGV : une controverse sur les compensations pour atteintes à la biodiversité, VertigO, 13, 2, doi: 10.4000/vertigo.14040.

Citation de l'article : Barataud F., Hellec F., Levain A., Petit S., 2018. Quand les recherches en sciences sociales s'invitent dans la gestion de l'eau. Nat. Sci. Soc. 26, 4, 395-406. 